Revista de la red interuniversitaria de estudios sobre las literaturas rioplatenses contemporáneas en Francia

$13 \mid 2015$

Nuevas experiencias editoriales y literaturas contemporáneas

\title{
Escrituras permeables : la autogestión editorial en la literatura. El caso de Gordo de Sagrado Sebakis y En construcción de Pablo Strucchi
}

Daniela Szpilbarg

\section{(2) OpenEdition}

Journals

Edición electrónica

URL: http://journals.openedition.org/lirico/2098

DOI: $10.4000 /$ lirico. 2098

ISSN: 2262-8339

Editor

Réseau interuniversitaire d'étude des littératures contemporaines du Río de la Plata

Referencia electrónica

Daniela Szpilbarg, «Escrituras permeables : la autogestión editorial en la literatura. El caso de Gordo de Sagrado Sebakis y En construcción de Pablo Strucchi », Cuadernos LIRICO [En línea], 13 | 2015, Puesto en línea el 15 diciembre 2015, consultado el 19 abril 2019. URL : http://journals.openedition.org/ lirico/2098 ; DOI : 10.4000/lirico.2098

Este documento fue generado automáticamente el 19 abril 2019.

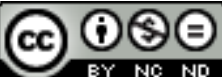

Cuadernos LIRICO está distribuido bajo una Licencia Creative Commons Atribución-NoComercialSinDerivar 4.0 Internacional. 


\title{
Escrituras permeables : la autogestión editorial en la literatura. El caso de Gordo de Sagrado Sebakis y En construcción de Pablo Strucchi
}

\author{
Daniela Szpilbarg
}

\section{Introducción}

1 La década del 2000 supuso, para el campo editorial en Argentina, la cristalización de transformaciones y reconfiguraciones que se habían gestado años antes. En parte como consecuencia de los procesos de racionalización, burocratización y concentración de editoriales producto de las fusiones, y en parte como consecuencia de los efectos de la crisis económica, política y social del año 2001. Cierre de librerías, nuevos canales de venta de los libros, fusiones empresariales que desnacionalizaron el sector (Botto 2006; Ruiz 2005), mostraron un modo de reorganización que giraba en torno a la necesidad de obtener rentabilidad. En paralelo, la edición nacional pequeña y mediana se reactivó a partir de la emergencia de editoriales que plantearon modos tradicionales pero también novedosos de pensar la (auto) gestión y la circulación de los libros.

En relación con esto, la hipótesis que pretende explorar este trabajo es que, si analizamos los procesos editoriales de la década del 2000, se puede establecer, en el polo de las pequeñas editoriales, una relación entre los materiales literarios, los procesos de edición y la generación de comunidades de lectura representadas en novelas que practican la autoficción como género característico ${ }^{1}$. En este sentido seguimos la afirmación de Pochettino (2010), quien propone pensar una relación homóloga entre los modos de la edición de libros y la enunciación presente en los materiales literarios. A esto debemos sumar la filiación editorial de ciertos autores que construyen una suerte de "comunidad" 
literario-editorial a partir de los vínculos de sociabilidad forjados en los años posteriores a la crisis de 2001. En este punto, y partiendo de los dos autores que hemos seleccionado para trabajar en este artículo, reflexionaremos sobre los procesos del campo editorial para vincular a los autores con la editorial que los publica.

En primer término, presentaremos algunas características de la década de 1990 y de los primeros años que siguieron a la crisis de 2001, para comprender los procesos neoliberales no solamente en su clásica interpretación "constrictiva" sino también, siguiendo a Gago (2014), vinculados con las vertientes de autoempresarialidad a las que las limitaciones del neoliberalismo y la ausencia del Estado dieron lugar. En este primer apartado ligaremos estos procesos al modo en que el campo editorial argentino se reconfiguró, reorganizándose en torno a dos o tres grandes emporios de la edición mundial que adquirieron editoriales locales y un enorme conjunto de pequeñas y medianas editoriales nacionales. En segundo término, describiremos la emergencia de la FLIA en el año 2006 como un evento que permitió cristalizar los acontecimientos ocurridos en los años anteriores alrededor del polo "autogestivo" de la edición, dando cuenta del surgimiento de emprendimientos microeditoriales "caseros" que comenzaron a encontrar espacios de circulación y difusión en el ámbito público. En relación con la emergencia de la FLIA, nos centraremos en el surgimiento de una editorial en particular, Milena Caserola, que si bien luego tuvo su derrotero convirtiéndose en una editorial más profesionalizada, comenzó en íntima relación con la Feria del Libro Independiente y sus principales organizadores. En el último apartado, intentaremos desarrollar la hipótesis de las literaturas "permeables" : una serie de textos de autores que trabajan sobre materiales autobiográficos para construir relatos autoficcionales en los que se da cuenta de una comunidad de lectura formada alrededor de la FLIA. A través de la puesta en juego de dinámicas de lo real-ficcional, esos relatos construyen esa comunidad. En este último apartado observaremos, en las novelas Gordo de Sagrado Sebakis y En construcción de Pablo Strucchi, algunas marcas que permiten pensar la sociabilidad en el ámbito literarioeditorial, así como también explorar la pregunta por el género de estas novelas que, si bien incluyen una cantidad de elementos autobiográficos, no constituyen autobiografías, sino que se vuelcan hacia la autoficción.

\section{Crisis y respuestas sociopolíticas : las dos caras del neoliberalismo}

4 Para situarnos en las transformaciones ocurridas en el campo editorial, es preciso contextualizar este sector dentro de la esfera de producción cultural. En los años '90 se produjeron cambios estructurales vinculados con el rol del Estado en la sociedad. Las políticas neoliberales implantadas por el ministro de Economía de Carlos Menem, Domingo Cavallo, impusieron una reforma del Estado cuyo punto principal fue la privatización de las empresas de servicios públicos y la decisión de no intervenir en intercambios comerciales (Botto 2006 ; Tenti 1993, Pucciarelli 2004). Esto posibilitó que las empresas extranjeras ingresaran al mercado. Hasta ese momento, si bien la trayectoria de la industria editorial local se había visto influenciada por movimientos de internacionalización, no había alcanzado tal grado de concentración. Los distintos sectores de actividad sufrieron cambios, principalmente la concentración y una tendencia a la extranjerización de la propiedad -las empresas extranjeras aumentaron mientras que las nacionales se redujeron entre 1991 y 2001 (Heredia 2011)-. La producción no era 
prioridad, por lo que las inversiones productivas eran escasas en relación a la especulación financiera.

5 Durante esta década quedó evidenciado que ante la irrupción del pensamiento neoliberal, se abrió una disputa importante entre el Estado, el mercado y los organismos internacionales para dirimir quién tenía el poder de decisión. Si bien suele hablarse de los efectos del neoliberalismo como reducidos a la década del noventa, Verónica Gago (2014) destaca que este proceso debe ser pensado más allá de ser un término "fijado en el pasado". Según esta autora, el neoliberalismo debe ser considerado -desde una perspectiva cercana a Foucault- como una "mutación en el arte de gobernar", que a través de saberes, tecnologías y prácticas despliega una racionalidad de nuevo tipo que no puede pensarse sólo como "impulsada desde arriba", sino que también tiene efectos en las formas de vida, resistencia y modos de habitar de los sectores populares. Con esto se refiere a que el neoliberalismo no solamente es un régimen de acumulación global que se observa en los modos de organización de las corporaciones económicas, sino que también es una racionalidad que "por abajo" negocia beneficios en contextos de desposesión (Gago $10-11)^{2}$.

6 Volviendo al mercado editorial de la década del 90, los cambios instaurados generaron efectos en la industria del libro en diferentes niveles : por un lado el sector editorial local se redujo (si tomamos como referencia el vuelco de editoriales locales a capitales extranjeros) ; por otro lado, esto incidió fuertemente en los hábitos de lectura del público y en el cierre de librerías (Ruiz 21). En consecuencia, los grupos multinacionales avanzaron en la expansión de los mercados editoriales liderados por inversores españoles, conformando grupos empresariales. Sin embargo, hay que destacar que esta concentración económica puede pensarse también, como hemos dicho, en el sentido de la emergencia de un "neoliberalismo desde abajo" (Gago 12) : un conjunto de condiciones que se concretan más allá de un gobierno en particular, pero que arrastran efectos en términos de una red de prácticas y saberes que pueden objetivarse en una economía popular. Esta economía mezcla saberes autogestivos con una "autoempresarialidad" que, en algunos sectores, provoca la organización de economías informales ${ }^{3}$. En el caso del campo editorial de los años 2000, esto puede observarse en emprendimientos editoriales comunitarios como Eloísa Cartonera o en la gestión de la FLIA como movimiento cultural, que desarrollaremos en los próximos apartados.

7 Con respecto a los efectos del neoliberalismo "desde arriba", diversos grupos comenzaron a constituirse marcando una nueva configuración del campo editorial. En primer lugar, el Grupo Planeta : entre 1998 y 2000, el grupo de Barcelona adquirió Emecé (en el año 2000), Paidós y Minotauro, entre las editoriales argentinas y Tusquets, Seix Barral, Ariel, Espasa-Calpe, Crítica, Temas de Hoy, Destino y Martínez Roca, entre las españolas (Ruiz 2005; De Diego 2006; Botto 2006; Seivach 2003). Emecé tenía una particularidad : por un lado le permitió a Planeta tener los derechos exclusivos de autores como Jorge Luis Borges y Adolfo Bioy Casares y, por otro lado, la traducción de autores de bestsellers como Sydney Sheldon, Robin Cook o Wilbur Smith, lo que le permitió dominar también la franja comercial de ficción internacional en castellano. Cabe destacar que el Grupo Planeta en la actualidad tiene más de 100 sellos en España y América Latina, 15000 autores, y publica 6500 títulos y 130 millones de libros por año ${ }^{4}$.

8 En segundo lugar, el Grupo Penguin RHM : el grupo Bertelsmann, que en 1997 había comprado Random House, se asoció en un 50 \% con Mondadori para la edición de libros en español. En 1998 compró el 60 \% de la editorial Sudamericana, segunda empresa del 
sector en ventas, sellando así la pérdida de competitividad de la industria editorial argentina frente a las transnacionales. Este gran grupo ya era propietario, en ese momento, de Random House, Grijalbo, Mondadori, Lumen, Debate y Plaza y Janés (De Diego 2006 ; Becerra, Hernández, Postolski 2003 ; Ruiz 2005). En el año 2001 se completó la operación de la venta de Sudamericana. Esta editorial había sido hegemónica en el campo editorial local ya que fue, entre otras cosas, la que editó por primera vez Cien años de Soledad, de Gabriel García Márquez, y todos los títulos posteriores del autor colombiano. Luego, en los últimos años, los procesos de concentración editorial continuaron, uniéndose Random House Mondadori a Penguin en el año 2013 y luego comprando al grupo Santillana (dueños del sello Alfaguara) en 2014. Otros grupos fueron el español Zeta, que tenía Bruguera y adquirió en 1996 Javier Vergara, el Grupo Prisa, que adquirió Santillana, Alfaguara, Taurus, Aguilar, Altea, y el Grupo Norma, de capitales colombianos, que adquirió Kapelusz en 1994. Estos grupos pasaron a ocupar las posiciones dominantes en el mercado editorial argentino concentrando la mayor parte de los beneficios.

9 En este contexto de concentración editorial (Botto 2006), y de un estado neoliberal (García Delgado 2003 ; Svampa 2005) emergió lo que algunos autores han caracterizado como una nueva cuestión social. Una parte de la población queda sumida en la pobreza, y con el transcurso de los primeros años poscrisis pudo observarse que a nivel social emergieron un conjunto de organizaciones que intentaron elaborar esta crisis de manera colectiva. Según Maristella Svampa (2005), una de las variables fundamentales que emergieron de la experiencia asamblearia, aun con sus contradicciones, fue la autonomía. Paradójicamente -afirma- al calor de las discusiones y fragmentaciones, lo que se fue precisando y cobrando espesor fue una demanda de autonomía que se constituyó en una variable de configuración política.

Este ethos militante y autogestivo se puso en práctica en una serie de colectivos culturales que se vieron potenciados a partir del 2001, como GAC, Grupo Etcétera, Cine Insurgente, así como colectivos de información alternativa, como La Vaca, Indymedia, Agencia Rodolfo Walsh, los cuales crearon nuevos sentidos políticos al mismo tiempo que asumieron el rol de comunicar los acontecimientos políticos y sociales. Estos proyectos encontraron lugares de canalización y de cruce con las experiencias de fábricas recuperadas, como la fábrica Brukman, IMPA o las imprentas recuperadas Chilavert y la Gráfica Patricios. Estos grupos se desarrollaron por relaciones de afinidad y encontraron su característica propia a partir de la acción directa y el rechazo a la mercantilización de la vida social. Al mismo tiempo, fueron vinculándose con otras luchas político-sociales, en el marco de relaciones de afinidad y solidaridad con otras organizaciones sociales movilizadas y movimientos sociales.

\section{La FLIA como concepción performática del mercado de libros}

11 Hay algunos proyectos que pueden pensarse como antecedentes de la emergencia de la FLIA. En esta línea se encuentra )el asunto(, un proyecto fundado y coordinado por Pablo Strucchi que surgió en el año 2001 : apareció como un espacio para la edición y difusión de libros sin constituirse como una clásica editorial con un catálogo. Este proyecto buscaba además agilizar el camino a los autores que quisieran publicar sus libros, así como facilitar la distribución de los libros a partir del encuentro entre el autor y el lector sin pasar por la figura del distribuidor y el espacio de la librería -espacio que 
representaba en ese momento el modo de producción editorial neoliberal, a partir de los procesos de marketing en los "puntos de venta"-.

12 Otro ejemplo es el proyecto editorial-social-productivo Eloísa Cartonera (surgido en 2003), que marcó una modalidad de construcción de lazos sociales en un momento de gran fragmentación y segregación social. Poniendo a circular un saber ligado a la autogestión, este conocimiento de un grupo de escritores y poetas cuajó con las condiciones de posibilidad y restricciones de la poscrisis del 2001, marcando nuevas formas de producción cultural no tradicionales ni corporativas (Pinkus y López 2007). Fue, según Marilina Winik, en los nuevos formatos de la organización política y cultural devenidas de la "generación post 2001" donde se recrearon "otras maneras de trabajar y poner en juego subjetividades no mercantilistas, afectivas y resistentes apoyadas en redes de trabajo" (145).

13 La emergencia de espacios como la Feria del Libro Independiente y Alternativo (FLIA) desarrollada en la Ciudad de Buenos Aires, y los distintos encuentros diseminados en todo el país, como la FLIA Cospel (Chaco), espacio Indygentes (Mendoza), Libros son (Córdoba), entre otros, favoreció procedimientos vinculares y de construcción dinámica de redes que desplegaron -como también afirma Winik- operaciones de resistencia y creación de comunidades alternativas de escritura, de difusión y circulación artístico-literaria. En este tipo de eventos y circuitos novedosos - que en la Ciudad de Buenos Aires se nucleaban en algunos espacios como el Centro Cultural Pachamama o La Tribu- cambió también la figura del escritor, que pasó a ser considerado como alguien que circulaba, vendía sus libros, estaba en contacto con lectores y editores y participaba del proceso de gestión editorial y distribución de sus obras. Como afirmaba Marilina Winik en una entrevista realizada en 2010 :

Lo que agrupó la FLIA en un comienzo fue ese underground literario. El under es una actitud política, querer mostrar o producir contenido y difundir autores. La idea del under está relacionada con un discurso y una posición en torno a cómo se hacen los libros. También el tema del copyleft, porque en esa modalidad se ve la circulación de los libros sin intermediaciones entre autor y lector. El copyleft tiene que ver con el deseo propio que manifiesta el autor respecto de qué hacer con la distribución de los libros (Szpilbarg, 2015) . $^{5}$ 


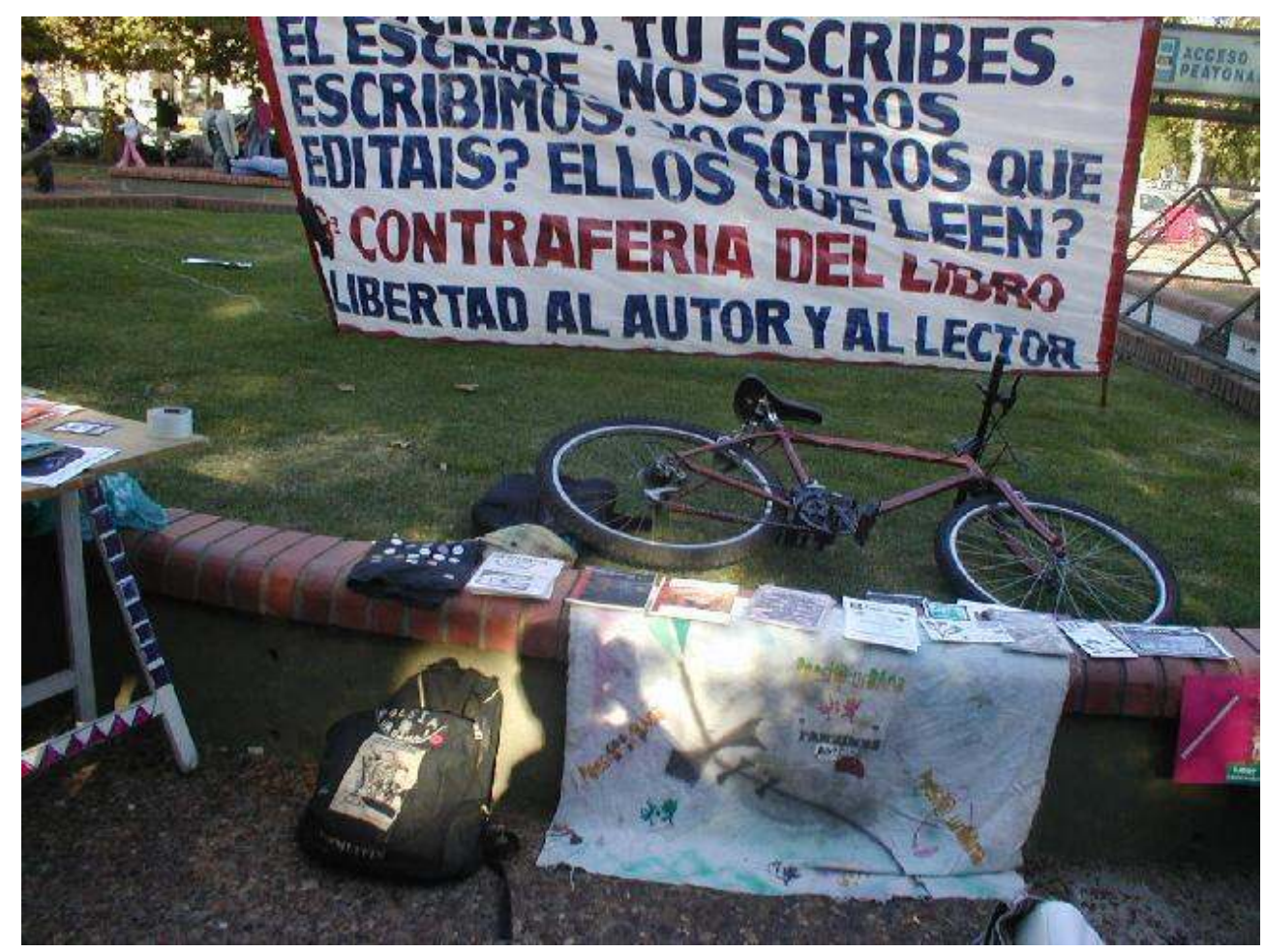

Contraferia del Libro en la Rural (2006) Fuente : Indymedia

14 En abril de 2006, durante la Feria del Libro de Buenos Aires, se autoconvocó un grupo de escritores independientes al costado de la fila para ingresar a la Feria del Libro. Los participantes principales eran los integrantes del grupo "Maldita Ginebra", "Poesía Urbana" y )el asunto(. Bajo un cartel que afirmaba "Yo escribo. Tú escribes. Él escribe. Nosotros escribimos. ¿ Vosotros qué editáis? ¿ Ellos qué leen? Contraferia del libro. Libertad al autor y al lector", se exponían los libros en una mesa. En una nota publicada en el portal Indymedia, se caracterizaba a la Feria del Libro como "el primer shopping donde hay que pagar entrada para consumir". Además se mencionaba que esa contraferia era una primera actividad rumbo al encuentro principal "Feria del libro independiente", que se proponía como el "cierre" de las actividades de la Contraferia. Allí también se promocionaban las reuniones organizativas que se realizaban en la Radio La Tribu y se convocaba de manera abierta tanto a las reuniones como a participar de la feria.

La protesta iba en tres direcciones: la contraferia se presentaba como antítesis de la mercantilización de los libros, de la privatización de los espacios culturales y de los parámetros de legitimación generada por la curaduría que interviene en el proceso editorial. Más allá de la idea de hacer la Contraferia en el mismo espacio (pero en el exterior) de la Feria del Libro de Buenos Aires, como una forma de "protesta", lo fundacional de la FLIA aparece en la voz de un escritor que preguntó por qué, en vez de hacer una contraferia, no hacían una feria propia, autónoma, con sus propios libros, "en otro lugar y en otro momento" que la Feria oficial. Finalmente, el 14 de mayo de 2006 se realizó la primera Feria del libro independiente en la Mutual Sentimiento, en el centro cultural Sexto Cultural. Los organizadores convocaron a José Luis Mangieri para dar una charla sobre la edición independiente en los 60 y los 70, marcando así que la Feria del Libro Independiente formaba parte de una tradición. La Feria se organizó en torno a dos ejes : gratuidad en el ingreso (contra la privatización) y libertad de puesto. Además, se descontaba que la intervención de colectivos editoriales como )el asunto( proponía un 
acceso a la publicación de libros de manera irrestricta. Como fruto de este espacio experimental de confluencia de formatos editoriales y estéticos, la FLIA emergió en la escena porteña, "(auto) construyéndose por afuera de las industrias culturales y los esponsoreos, como un acontecimiento que se territorializaba y desterritorializaba cada vez, creando así un hiato para escritores, editoriales y proyectos sociales donde se permitía exhibir y distribuir libros" (Winik 2010). El evento, según Winik, derivó por territorios en disputa, como fábricas recuperadas, universidades y asambleas barriales como puede observarse en algunos de los afiches publicitarios-, constituyendo un acontecimiento resistente tanto por su capacidad organizativa, como por su capacidad de crear audiencias que participaban del evento, visibilizando el copyleft como una práctica que fomentaba el libre acceso y distribución de materiales textuales, teniendo en cuenta que en las últimas décadas la digitalización había puesto en jaque inversiones de las industrias culturales, convirtiendo al copyright en una suerte de "policía cultural" (Winik 2010). El devenir técnico y tecnológico (específicamente la digitalización, que permitió entre otras cosas transmitir y distribuir materiales sin costo), suprimió "la distinción entre original y copia" (Winik 2010), lo cual fue propiciando condiciones de intercambio de libros y textos que contribuyeron a generar un nuevo circuito editorial alternativo a los del mainstream. 


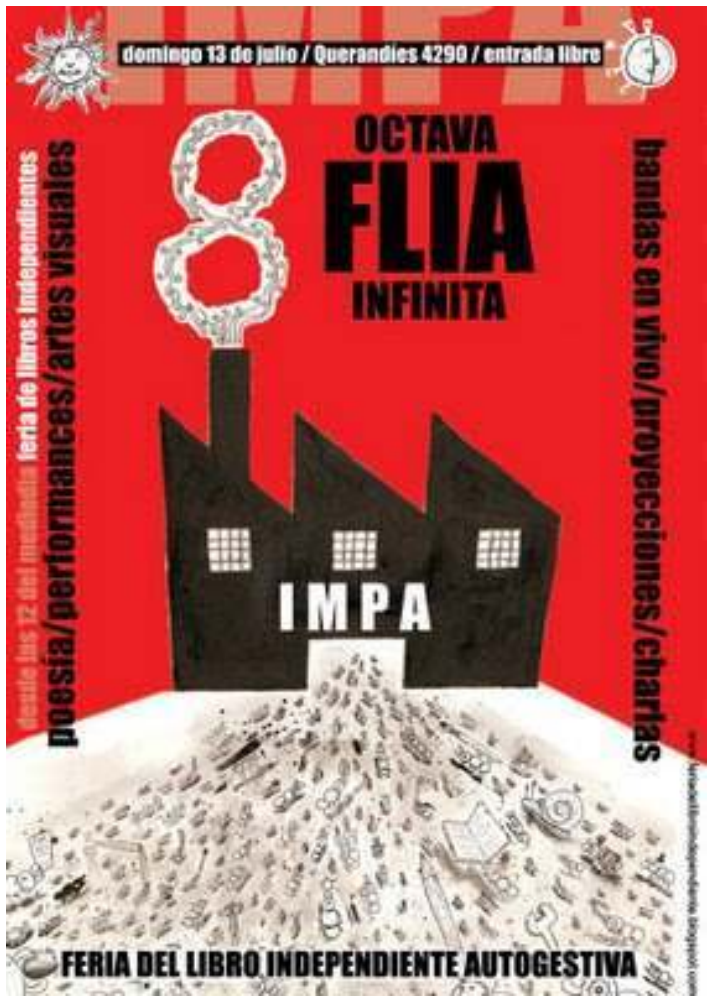

\section{DOMINGO 29 DE MARZO DE 12 A 23 HS.}
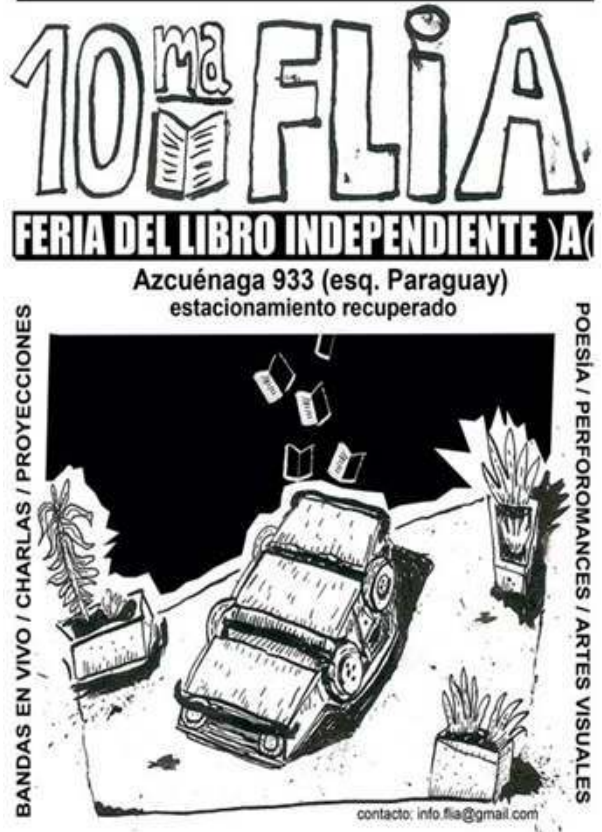

LAFLIA SE ESTACIONA EN SOCIALES

www.feriadellibroindependiente.blogspot.com 
FLIA en el estacionamiento de la Facultad de Filosofía y Letras. (2011)

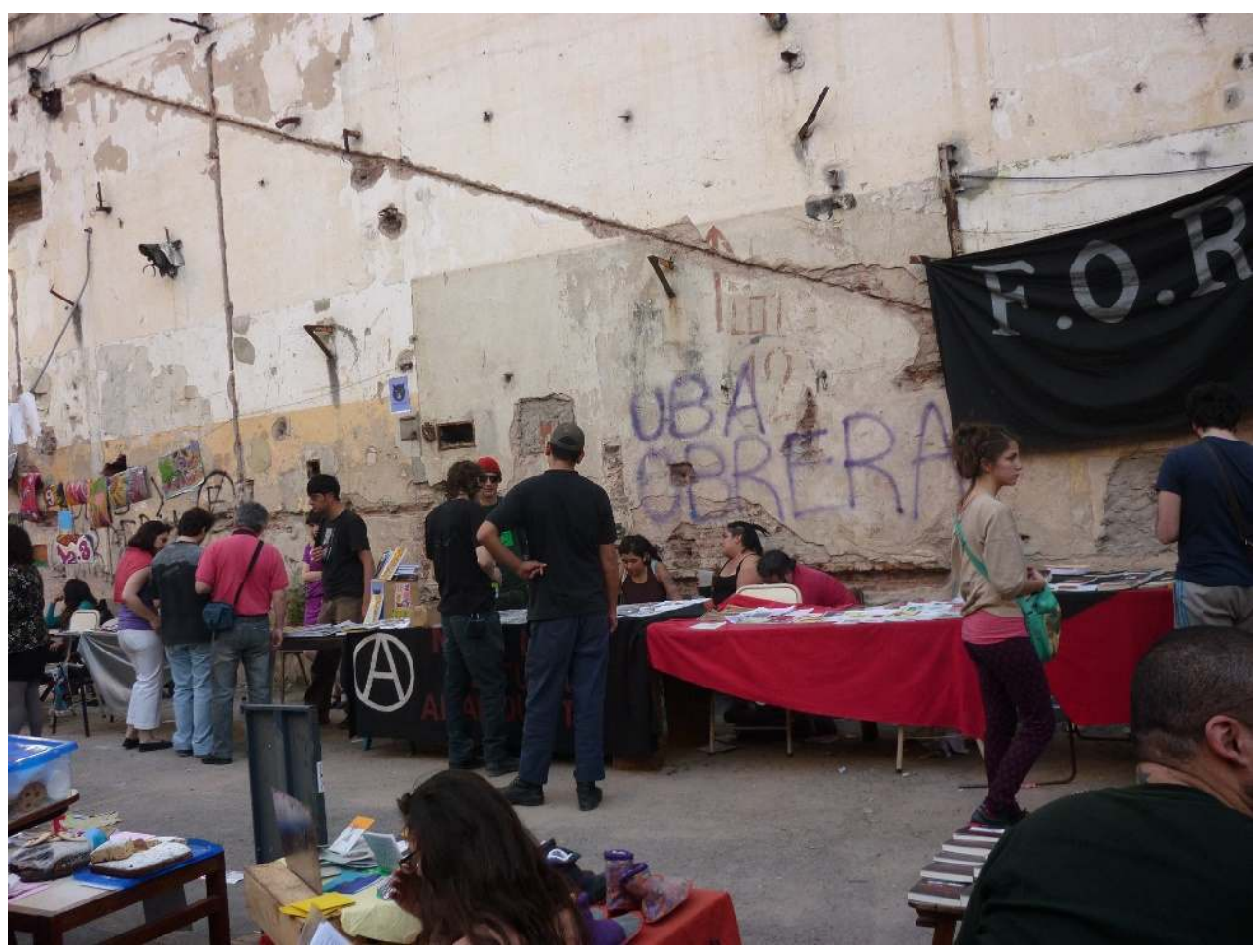

\section{La editorial Milena Caserola como proyecto experiencial}

El nombre de una editorial es siempre significativo : indicio de la construcción de una identidad que debe además ser interpretado a la luz del contexto histórico; marca indeleble que nos permite muchas veces situar y pensar la editorial "ajustada a las disputas de una época" (Sorá 1997). Milena Caserola contiene y representa desde su mismo nombre algunos de estos avatares, por lo que el nombre se transforma en un emergente que, en el acto de fundación/nominación encarna procesos políticos de autogestión ejerciendo una resistencia desde las prácticas para pensar la circulación de los libros.

A fin de vincularla con la organización de la FLIA y con los casos de análisis que hemos abordado, lo primero que podemos afirmar es que la emergencia de Milena Caserola fue un acontecimiento colectivo. Decimos que fue un acontecimiento porque la emergencia de la editorial se enraíza en un evento en particular. En ese evento, ocurrido en ocasión de la primera publicación de la editorial, la presentación y puesta en circulación del libro adquirieron un carácter performático. La historia de este primer libro que sale con el sello Milena Caserola es significativa y condensa la postura ideológica representada en el objetivo de su editor, Matías Reck, de "subvertir ciertos valores dentro del campo editorial", vinculados principalmente con la mercantilización de los libros. Este primer libro era un texto de Giorgio Agamben llamado Idea de la prosa. Editado de manera "pirata", la existencia de internet permitió "bajar" el texto, que estaba agotado. Además de editarlo, los integrantes de la editorial realizaron un acto editorial disruptivo. Fueron a la conferencia donde exponía Agamben (editado por Adriana Hidalgo), y pusieron el libro a la venta a diez pesos. Reck refiere la situación de esta manera : 
En la primera conferencia casi nos echaron, porque estábamos vendiendo libros en una conferencia en la que no se podía y porque además estaban los libros de Adriana Hidalgo que eran los que tenían los derechos de la obra de Agamben. Nosotros nos divertimos, vendimos libros, y creo que el acto editorial que hicimos estuvo bueno porque devolvimos un libro al mercado. En otra conferencia, una mujer compró uno de los libros y se lo hizo firmar a Agamben. Ahí nos tuvimos que ir corriendo: fue una cierta aventura juvenil de jugar con la cuestión de los derechos de autor y del editor. De alguna manera esa primera experiencia editorial sobre un libro terminó siendo una editorial. (Szpilbarg, 2015)

Luego de este primer evento, Reck comenzó a editar autores que conocía y que querían publicar sus libros. Eran amigos y otros escritores y poetas que conocía en las FLIAs. Podemos afirmar entonces que la edición se fue configurando mediada por lazos de confianza y amistad entre el autor y el editor. También hay otro elemento fundamental que es preciso desarrollar para dar cuenta de la trayectoria de la editorial y de la conformación de comunidades editoriales-literarias: la historia de esta editorial está indisociablemente unida a la emergencia de la FLIA; es en este marco que la editorial comienza a construir su catálogo, su posicionamiento en el campo de la edición y sus modalidades de circulación y distribución características como parte de este "colectivo de colectivos" que representaba la feria. Es importante destacar que los modos de circulación y distribución de los libros son tres canales : la presentación del libro, la venta en ferias y festivales en Buenos Aires y otras ciudades del interior, y en tercer lugar la distribución del $20 \%$ de la tirada a través de librerías, con la mediación de una distribuidora. Estos datos nos muestran que se trata de una editorial que sostiene su circulación principalmente en ámbitos informales donde el componente afectivo y cara a cara es una de las características sobresalientes. Se trata de una venta "dirigida" y muchas veces "específica" que reafirma también la importancia de la sociabilidad en este proyecto editorial y en la relación que une al editor con los autores.

\section{La comunidad en la escritura a través de la autoficción. Los casos de Gordo y En construcción}

19 Hemos seleccionado en este apartado dos novelas que nos permiten pensar los vínculos entre literatura y edición a partir de la historia narrada: Gordo, de Sagrado Sebakis, editada en 2011, y En construcción de Pablo Strucchi, editada en 2014. En ambas novelas puede notarse, apenas comenzada la lectura, la presencia de un narrador homodiegético que presenta los eventos de la historia de un modo hiperrealista en el que abundan las marcas de contemporaneidad. El yo es central, y los personajes trazan una combinación de ficcionalización de personas reales y una condensación, en personajes con nombre ficticio, de personas que habitan el mundo real de estos autores. Por otro lado, conviven en las novelas personajes ficcionales y nombres de personas y escritores reales, lo cual permite abonar la hipótesis de la autoficción que construyen a partir de esta contradicción entre la novela tradicional y la autobiografía, oscilando entre los dos polos, pero sin posarse en ninguno.

20 Gordo de Sagrado Sebakis está compuesta por tres textos breves -Risperidona, Sertindól y Zyprexa- que retratan la vida cotidiana de un escritor joven del under literario, tanto sus actividades y trabajos, como sus consumos privados. En primer lugar, podemos observar que hay un juego sobre la identidad partiendo de la base de los elementos paratextuales : la tapa, la solapa y la contratapa del libro. La novela no tiene ningún nombre de autor en 
la tapa, y cuando la abrimos observamos que está escrita por "Sagrado Sebakis", lo que parece ser un seudónimo. A partir de allí hay una suerte de narración -como afirma Incardona en el prólogo- de "una identidad que estará atravesada por la figura de autor hasta romperse, esquizofrénica". Hay referencias constantes a personajes del circuito literario y editorial en el cual este personaje-narrador-escritor construye sus experiencias cotidianas (viajes, encuentros, congresos, lecturas, reuniones, charlas con amigos); esas experiencias aparecen combinadas o alternadas con sus consumos en un universo digital - ¿real, ficcional ?-, en el cual también se muestran los modos de relación propios del paradigma electrónico. Gordo es inseparable de Internet y la aparición de las redes sociales; Sadier y Picotto (2012) sostienen que "es un libro hecho en Internet, que no funciona sin Internet, ya sea porque es leído por el autor en un video posteado en Facebook, o bien porque se puede 'bajar' de la página de la distribuidora con un código QR " (Sadier y Picotto 2012).

21 La novela En construcción de Pablo Strucchi está narrada en primera persona. La acción comienza en el año 2001, aunque en la novela se alternan épocas y se narran distintos momentos significativos : 2006, 2001, 2003, al mismo tiempo que el narrador se centra en la descripción y las historias de diferentes personajes del mundo literario y del mundo de la construcción. El narrador, que comparte marcas identitarias con el mismo Strucchi, como por ejemplo su doble dedicación a la actividad de la construcción y a su labor como activista cultural, cuenta su experiencia como trabajador en el mundo de la construcción, mientras va desplegando el relato de los eventos sociales y culturales -relacionados a la FLIA y a colectivos culturales- que lo tuvieron como participante en los últimos años. Allí se observa entonces la coexistencia de su biografía junto con los eventos más significativos que, aunque no están narrados cronológicamente, permiten reconstruir la conformación de un grupo social nucleado alrededor de la edición y circulación de libros. También aparecen en la novela reflexiones y cuestionamientos acerca de la edición contemporánea, que pueden leerse como una toma de posición de parte del autor/ narrador/personaje.

Como parte de esta autoficción, en su relato se mezclan personajes ficcionales con reales :

Todos los sábados a mediodía en La Paternal te podías encontrar con los más grandes poetas, desde Sebastián Kirzner hasta Liliana Lukin, desde Héctor Urruspuru a Gabriela Bejerman, desde Edwards a Javito. (Strucchi, $2011: 62$ )

23 Ambos personajes-narradores son escritores y se erigen como integrantes de un grupo : una comunidad "literaria" conformada por poetas, escritores, artistas, y un editor al que se reconoce como una figura que nuclea y contiene a estos personajes. Este editor aparece con el nombre real de Reck (editor de Milena Caserola) en el caso de Gordo, y está ficcionalizado en el caso de En construcción, en donde aparece también un personaje editor pero que podría ser a un tiempo distintos personajes reales, desde el mismo Strucchi hasta Reck, o incluso podría pensarse que Strucchi ficcionaliza la figura del editor para volverlo una figura sin anclaje real que lo ligue a una única persona. En ambos casos se describe a este editor como una suerte de "contenedor" de los autores que edita; y en ambos casos los lazos de amistad y confianza entre los escritores/narradores y el editor y el proyecto editorial son evidentes :

El PH en el que vivo perteneció originalmente a Matías Reck, el Luke Skywalker psicosomático del circuito independiente, gran amigo y sabueso editorial [...] hace un tiempo viajó a Alemania para presentar nuestra editorial en la Feria del Libro de Frankfurt, el circo literario más grande del mundo.[...]. Abrió literalmente una 
bandera okupa dentro de la Feria apostando a hacer visible entre otras cosas, el asuntillo ese del copyleft. (Sagrado Sebakis, $2011: 129$ ) que se relaciona con los poetas en un vínculo de mutua necesidad, pero también de amistad, contención y afecto :

Los Poetas pensaban que lo que hacía Sebas [el editor] era un servicio a la comunidad. Es más, ni siquiera pensaban, no se preguntaban cómo había llegado ahí una imprenta para que usen gratuitamente, pero si la imprenta estaba ahí, había que usarla, y si se trababa la impresora había que destrabarla y Sebas era el que sabía, el que llevaba los números, el que administraba la cooperativa, el que compraba el papel, el que cobraba, el que barría y los Poetas caían con sus birras y sus porros a imprimir y a juntarse con otros Poetas a ver cómo es que lograba hacer de una idea un libro... (Strucchi, $2011: 150$ )

Su función es quedar embarazada de libros que engendran otros, esos otros que apenas saben cómo se procrea, o que quizás son expertas, que son capaces de copular veinticuatro horas seguidas pero apenas pueden levantarse a las siete de la mañana tres días al hilo porque el niño demanda tiempo. Ni hablar de darle la teta cuando llora. Y ahí está Sebas, no solo editando el libro sino amamantando a la mamá. (Strucchi, $2011: 151$ )

En los casos analizados, el yo autoficcional se inscribe en relación con otros. Pochettino (2011) se pregunta entonces qué comunidades advienen en esta inscripción, y de qué modo la literatura reciente asume la autofiguración de un yo-entre-amigos, en el límite de una comunidad de escritura. Y sostiene que este tipo de publicaciones, tanto de estos autores como de otros participantes de la FLIA, reconocen el lugar disidente de todo el proceso de creación y circulación de sus textos, lo cual puede observarse desde la misma "página de legales", en la cual se observan aspectos subjetivos y posiciones tomadas respecto de la comunidad editorial/literaria de pertenencia, así como la postura respecto de la liberación de los contenidos del libro. Estas posturas, que pueden observarse en los libros de Milena Caserola con una leyenda fija ${ }^{6}$, así como en algunos libros publicados por el colectivo )el asunto(, interpelan al lector acerca del sentido de las posturas ligadas al copyleft. En el caso de Gordo, además, notamos que en la contratapa aparece un código QR y que en la solapa se explica: "Este libro viene con un código $Q R$. Si tenés scan en tu celular, te lo podés robar en PDF". En consonancia con este juego vinculado a los derechos del autor, el libro está escrito con un seudónimo, y en la primera solapa pueden leerse una serie de datos falsos acerca del supuesto "autor", que ilustran una foto real de Sebastián Kirzner :

Sagrado Sebakis es argentino. Nació el 26 de noviembre de 1909 y falleció el 26 de noviembre de 2002. Y volvió en febrero de 2003. Esta es su cuarta reencarnación y GORDO la primera novela de este periodo. (Sagrado Sebakis, 2011)

Estos elementos muestran que el análisis de textos como estos exige abordar aspectos paratextuales en mucha mayor medida que en el caso de otros libros, en los que lo editorial, en el sentido institucional, está más disociado del texto literario. Ubicados por fuera de los canales tradicionales de la industria, la escritura no es para estos escritores una actividad profesional en el sentido de que representa su sostén material, por lo que puede observarse que en el tipo de autores publicados por editoriales pequeñas que editan sus primeras obras, o bien a través de procesos de autoedición, la importancia radica en visibilizarse y pasar a formar parte del campo literario. Esto puede observarse si tenemos en cuenta que algunos de los autores publicados por esta editorial han circulado,

Cuadernos LIRICO, 13 | 2015 
en años posteriores, por otras editoriales, como Enzo Maqueira (publicado luego por Interzona), o Gonzalo Unamuno (publicado por Galerna).

Volviendo a lo anterior, las novelas analizadas pueden leerse, desde lo paratextual a lo textual, como un mapa de afectividades: podemos encontrar en ellas aspectos que refieren a la inscripción y a la relación con la FLIA como espacio de sociabilidad pero también, en el caso de Strucchi, como circuito de militancia cultural. En ambos casos está presente en la construcción de un universo real-ficcional relativamente endogámico organizado en torno a la FLIA, que se visibiliza en el proceso de creación del libro. De esta manera, al tomar ciertos autores que practican la literatura autoficcional, se puede leer en ellos "procesos de autogestión, autopublicación y construcción de redes de proyectos de trabajo y gestión cultural, y problematizar la autobiografía como espacio de organización, producción de archivo, territorio de disputa y de visibilización de redes estéticas, políticas y editoriales" (Pochettino 2011 2). Hay un uso de la tradición de la escritura íntima pero "no como una práctica de introspección" (Blanckeman 2015), sino para invertir sus fundamentos. Se registran escenas vividas, en relación con los espacios, los lugares, la ciudad, abriendo así, como plantea Blackeman, una dimensión antropológica del relato ficcional, que lo transforma en una suerte de "etnotexto".

Esta modalidad, que marca así una permeabilidad entre realidad y ficción, postula nuevas formas de realismo y de constitución de la figura del autor y el editor en el marco de textos dirigidos a una red de sociabilidades que se construye y re-construye en el espacio tanto literario como extra-literario en una suerte de mutua articulación transformadora.

\section{Conclusiones}

En este trabajo hemos intentado observar, a partir de dos novelas de autores contemporáneos publicados por una editorial en una posición específica del campo editorial, tanto las relaciones que se tejen entre comunidades literarias y editoriales como el género en que estos autores practican su ficción. Así, hemos destacado la importancia de la autoficción, neologismo que designa una síntesis entre "lo autobiográfico y lo ficcional en una aparente contradicción" (Alberca 5), y en la cual se manifiesta una hibridación y una mezcla de géneros. Hemos visto, también, cómo esas relaciones entre comunidades se enmarcan en un circuito alternativo de difusión de literatura que comenzó a constituirse luego de la crisis del 2001, y que cristalizó en el año 2006 con el comienzo de la Feria del Libro Independiente y Alternativa. En estas narraciones autoficcionales, se representan "procesos de autogestión, autopublicación y construcción de redes de proyectos de trabajo y gestión cultural" (Pochettino 2011 1). A su vez, esas redes participan de planteos por la liberación de derechos, o por la creación de ferias de libros alternativas a las existentes. Así, los textos funcionan como un artefacto "que al tiempo que inscribe, promete acciones" (ibíd.). La edición se convierte en un "dispositivo complejo que hace visibles un estadio de autocrítica y anticipos de intervenciones" (ibíd. 5).

Otra de las conclusiones que quisiéramos expresar sigue la hipótesis de que la editorial aparece, en este contexto poscrisis, como un espacio nucleador de textos y personas que constituyen comunidades extra-literarias organizadas en torno a comunidades afectivas y militantes en un contexto histórico específico, del cual los libros son una suerte de "documento". 


\section{BIBLIOGRAFÍA}

Bibliografía

Alberca, Manuel “¿Existe la autoficción hispanoamericana?”, Cuadernos del CILHA. Número 7. Madrid, 2005.

Becerra, Martín, Hernández, Pablo, y Glenn Postolsky. "La Concentración de Las Industrias Culturales", Industrias culturales : mercado y políticas públicas en Argentina. Buenos Aires : Secretaría de Cultura de la Nación y Ciccus, 2003.

Blanckeman, Bruno. "Dossier". En Ernaux, Annie Diario del afuera/La vida exterior. Milena Caserola : Buenos Aires, 2015.

Bosoer, Fabián y Leiras Santiago "Posguerra fría, 'neodecisionismo' y nueva fase del capitalismo : el alegato del Príncipe-gobernante en el escenario global de los '90", en Borón, Atilio, Gambina, Julio y Naúm Minsburg (compiladores). Tiempos Violentos. Neoliberalismo, globalización y desigualdad en América Latina. Buenos Aires : Eudeba-Clacso, 1999.

Botto, Malena. "1990-2000. La concentración y la polarización de la industria editorial", en De Diego, José Luis (director). Editores y políticas editoriales en Argentina, 1880-2000. Buenos Aires : FCE, 2006.

Busaniche, Beatriz. Argentina copyleft. La crisis del modelo de derecho de autor y las prácticas para democratizar la cultura. Buenos Aires : Ediciones Boll Cono Sur, 2010.

Colleu, Gilles. La edición independiente como herramienta protagónica de la bibliodiversidad. Buenos aires : La marca Editora, 2008.

De Diego, José Luis (comp), Editores y políticas editoriales en Argentina 1880-2000, Buenos Aires, FCE (2006).

De Diego, José Luis “Concentración, económica, nuevos editores, nuevos agentes”, en Actas del Coloquio Argentino de Estudios sobre el Libro y la Edición. Facultad de Humanidades de la UNLP. Edición digital : http://coloquiolibroyedicion.fahce.unlp.edu.ar/actas (2012).

Delgado, Verónica y Espósito, Fabio “La emergencia del editor moderno”, en de Diego, José Luis (dir.). Editores y políticas editoriales en Argentina (1880-2000). Buenos Aires : Fondo de Cultura Económica, 2006.

Gago, Verónica. La razón neoliberal. Buenos Aires : Tinta Limón, 2014.

García Delgado, Daniel. Estado- Nación y la crisis del modelo. Buenos Aires : Editorial Vitral, 2003.

Heredia, Mariana. "La hechura de la política económica : los economistas, la convertibilidad y el modelo neoliberal”, en Pucciarelli, Alfredo (comp.). Los años de Menem. La construcción del orden neoliberal. Buenos Aires : Siglo XXI, 2011.

Jenny, Laurent, “L'autofiction” : https://www.unige.ch/lettres/framo/enseignements/ methodes/autofiction/afintegr.html (consultado el 02/11/2014).

Logan, Robert “¿Qué es un libro ? ¡Pasado, presente y futuro! De la tabla de arcilla al smartbook”, en Carlon, M. y Scolari, O (comp). El fin de los medios masivos. Buenos Aires : La crujía, 2009. 
Mazzoni Ana y Selci, Damián. “Poesía actual y cualquierización”. El interpretador. Buenos Aires, 2006. Disponible online : http://www.elinterpretador.net/26AnaMazzoniYDamianSelciPoesiaActualYCualquierizacion.html.

Pinkus y López. “Eloísa Cartonera : la subversión suave ante la crisis”, en Ledesma, María y Siganevich, Paula. Piquete de Ojo. Visualidades de la crisis. Buenos Aires : Ediciones FADU/Nobuko, 2007.

Pochettino, Anahí "Crisis, canon y comunidad en la literatura argentina reciente : programas estéticos, modos de asociación literaria-editorial y autobiografías colectivas". En III Jornadas de Investigación del Área Letras, Córdoba, 2012.

Pochettino, Anahí. "Modos de asociación literaria-editorial en textos autobiográficos de la literatura argentina reciente", en Recial. Revista del Ciffhyh, Area de Letras 3. Córdoba : Universidad Nacional de Córdoba, 2012.

Pucciarelli, Alfredo. Los años de Menem. La construcción del orden neoliberal. Buenos Aires : Siglo XXI, 2011.

Ruiz, Laura. Voces ásperas. Buenos Aires : Biblos, 2005.

Sadier, Emilio y Picotto, Diego. “En busca de la literatura blanca”. Lobo Suelto : http:// anarquiacoronada.blogspot.com.ar/2012/05/en-busca-de-la-literatura-blanca.html (consultado el 01/05/2012).

Saferstein, Ezequiel y Szpilbarg, Daniela. "La 'Independencia' en el espacio editorial porteño." En Wortman, Ana (comp.). Mi Buenos Aires querido. Entre la democratización cultural y la desigualdad educativa. Buenos Aires : Prometeo, 2012.

Sebakis, Sagrado. Gordo. Milena Caserola : Buenos Aires, 2011.

Sorá, Gustavo. "Da Casa à Empresa : A Nação e a Internacionalização nas Transformações do Campo Editorial no Brasil", ANPOCS, San Pablo (1997).

Strucchi, Pablo. En construcción. Buenos Aires : Milena Caserola, 2011.

Svampa, Maristella La sociedad excluyente. La Argentina bajo el signo del neoliberalismo, Buenos Aires, Taurus (2005)

Szpilbarg, Daniela "La vuelta al libro. Representaciones de editores artesanales sobre la industria del libro" en Revista Afuera. Estudios de crítica cultural, Buenos Aires (2010).

Szpilbarg, Daniela (2015) Las tramas de la edición mundializada. Transformaciones y horizontes del campo editorial en Argentina 1998-2013, (Tesis no publicada).

Szpilbarg, Daniela "Editores artesanales : sus modos de producción, circulación y consumo del libro en el campo editorial contemporáneo” en Páginas de Guarda 13 ; Buenos Aires. (2012).

Szpilbarg, Daniela y Saferstein, Ezequiel “La industria editorial argentina 1990-2010 : entre la concentración económica y la bibliodiversidad" en Alter/nativas Latin American Cultural Studies Journal Volumen 3, Ohio, Estados Unidos (2014).

Vanoli, Hernán "Pequeñas editoriales y transformaciones en la cultura literaria argentina". Apuntes de Investigación. Número 15. Buenos Aires, disponible en : http:// www.apuntescecyp.com.ar/index.php/apuntes/article/viewArticle/67 (consultado el 03/12/2014).

Vanoli, Hernán. Por una sociología del espacio editorial. Cuatro modelos de edición literaria en Argentina en el siglo XX. Buenos Aires : Facultad de Ciencias Sociales, UBA (tesis de Maestría no publicada, 2010. 
Vanoli, Hernan y Saferstein, Ezequiel. "Cultura literaria e industria editorial. Desencuentros, convergencias y preguntas alrededor de la escena de las pequeñas editoriales" en Paula Miguel (comp.). Emprendedores 0110 : Creatividad, economía y cultura en la ciudad de Buenos Aires 2001-2010. Buenos Aires : Aurelia Rivera, 2012.

Vigna, Diego. "Literatura y soportes digitales : La irrupción del blog en el sistema literario actual del país". Ponencia presentada en XII Jornadas Nacionales de Investigadores en Comunicación : "Nuevos escenarios y lenguajes convergentes". Rosario, 2008.

Winik, Marilina. "Ediciones copyleft", en Busaniche, Beatriz (editora). Argentina Copyleft. La crisis del modelo de derecho de autor y las prácticas para democratizar la cultura. Buenos Aires : Ediciones Boll Cono Sur, 2010.

Winik, Marilina, y Reck, Matías. "Un posible final para un certero inicio : acerca de los desafíos de las editoriales independientes." Ponencia presentada en Primer Coloquio Argentino de Estudios sobre el Libro y la Edición. La Plata : UNLP, 2012.

Wortman, Ana. Entre la política y la gestión de la cultura y el arte. Buenos Aires : EUDEBA, 2009.

\section{NOTAS}

1. "La autoficción aparece como un desvío ficcional de la autobiografía. Pero según un primer tipo de definición - estilístico-, la metamorfosis de la autobiografía en autoficción se funda en ciertos efectos que derivan del tipo del lenguaje empleado. Según un segundo tipo de definición -referencial-, la autobiografía se transforma en autoficción en función de su contenido, y de la relación del contenido con la realidad [...] El autor de la autoficción, a la vez que afirma que lo que cuenta es verdadero, advierte al lector contra una adhesión a esta creencia. Entonces, todos los elementos del relato pivotan entre valor factual y valor ficcional, sin que el lector pueda zanjar entre los dos" (Jenny 2003). Por otro lado, en el caso particular de estos autores, consideramos además que la sociabilidad literaria y editorial es tan significativa que se vuelve un material literario ineludible a la hora de construir sus novelas, las cuales, además, se vuelven una suerte de "documento histórico" para narrar una época de la cultura y contracultura argentinas.

2. Gago propone pensar el neoliberalismo no sólo como una lógica de gobierno, sino como una "forma anclada en los territorios" (14) que se manifiesta y articula con subjetividades específicas.

3. Para Gago, hablar de neoliberalismo "desde abajo" es un modo de dar cuenta de la dinámica que resiste a la explotación y la desposesión y que a la vez se despliega en territorios, fortalecida en las subjetividades populares y proliferante en términos expansivos en las economías informales. Esto supone no pensar que el neoliberalismo es solamente un conjunto de macropolíticas diseñadas por centros imperialistas, ni tampoco suponer que como racionalidad compete solamente a grandes actores políticos y económicos, sean regionales, transnacionales o locales, sino pensar el nivel molecular en que la racionalidad neoliberal se ha expandido.

4. Datos extraídos de la página web de Planeta en el año 2014, www.planeta.es.

5. Los cambios tecnológicos ejercen un rol central a la hora de definir las políticas y sistemas jurídicos que determinan las formas de producción, distribución y acceso a la cultura en los diferentes momentos históricos. El término copyleft (que empezó a utilizarse en la década del 2000), refiere a una práctica vinculada a las comunidades de software libre ; surgió como un juego de palabras para marcar la idea opuesta al copyright o derecho de autor. El copyleft surge, además, de las posibilidades que la digitalización supuso a la hora de pensar las tensiones de la "propiedad intelectual" (Busaniche 2010). A través de la práctica del copyleft y de las licencias libres, se propone al autor de una obra literaria o de otro tipo que "libere" el contenido de su obra para 
que pueda ser copiado, reproducido y compartido. Se desarrolla en oposición a las prácticas tradicionales de la industria del libro.

6. En la página de legales de los libros de Milena Caserola aparece la leyenda "Todos los izquierdos están reservados, si no remítanse a la lista de libros censurados en las distintas dictaduras y democracias. Por lo que privar a alguien de quemar un libro a la luz de la fotocopiadora es promover la desaparición de lectores."

\section{RESÚMENES}

Dentro de los eventos más significativos en el mundo editorial argentino de los años 2000, podemos destacar el surgimiento de la FLIA (Feria del Libro Independiente y Alternativa) en 2006. Este evento (vinculado a la cultura asamblearia derivada de los cambios políticos, económicos y sociales causados por la crisis de diciembre de 2001, pero también a la constricción editorial existente en aquel momento) supuso el desarrollo de un mercado literario informal caracterizado por una serie de editoriales autogestionadas que construyeron sus propias dinámicas de distribución, moldeando las comunidades de lectura y escritura que alimentaban esos mismos proyectos editoriales. En este artículo tomaremos como caso de análisis la editorial Milena Caserola (surgida en 2005) y su editor Matías Reck -quien fuera uno de los organizadores de la primera FLIA-, para analizar, en primer lugar, una serie de editoriales que funcionan en los márgenes del campo. Por último, observaremos, en un corpus de dos novelas de autores muy vinculados con la FLIA, los modos en que la experiencia editorial se inscribe en las narrativas y qué tipo de relaciones se construyen entre las figuras de autor-editor-público.

Parmi les changements les plus importants dans le monde de l'édition argentine des années 2000, l'émergence de FLIA (Salon du livre indépendant et alternatif) en 2006 constitue un événement majeur. Liée à la culture des assemblées populaires issue des transformations politiques, économiques et sociales provoquées par la crise de décembre 2001, mais aussi aux contraintes auxquelles se heurtait le monde de l'édition à la même époque, la FLIA a contribué au développement d'un marché littéraire informel caractérisé par une série d'éditeurs autogérés ayant construit leur propre dynamique de distribution, commercialisation et consommation, ainsi que leurs propres communautés de lecture et d'écriture. Dans cet article, nous abordons le cas de la maison d'édition Milena Caserola (créée en 2005) et de son éditeur Matías Reck -un des organisateurs de la première FLIA- afin d'analyser, en premier lieu, les dynamiques propres à un certain nombre d'éditeurs qui fonctionnent dans les marges du champ éditorial. En deuxième lieu, nous examinons, dans un corpus de deux romans écrits par des " auteurs FLIA », comment cette nouvelle expérience éditoriale est décelable dans le niveau textuel en mettant l'accent sur les relations entre la figure de l'auteur, de l'éditeur et du publique.

Among the most significant changes in the Argentine publishing field of the 2000, the emergence of FLIA (Independent and Alternative Book Fair) in 2006 was a major event. Linked to the culture of the assembly derived from the political, economic, and social changes caused by the crisis of December 2001, but also to the publishing constrictions at the time, the FLIA involved the development of an informal literary market characterized by a series of self-managed publishers who built their own distribution dynamics, creating literacy communities nourished by the same publishing projects. In this article, we will focus on the publishing house Milena Caserola 
(created in 2005) and its editor, Matías Reck - who was one of the organizers of the first FLIA-, to analyse, in the first place, the dynamics of a number of publishers operating in the margins of the field. In the second place, we will examine, in a corpus of two novels by authors closely linked with the FLIA, the ways in which the publishing experience intervenes into narratives. We will also try to determine what kinds of relationships are built between the figures of author, publisher, and public.

ÍNDICE

Mots-clés: sociabilité, Salon du livre indépendant (FLIA), champ éditorial, éditeurs autogérés Palabras claves: campo editorial, Feria del Libro Independiente y Alternativa (FLIA), editores autogestionados, sociabilidad

Keywords: publishing field, Argentina, Independent Book Fair (FLIA), sociability, self-managed publishers

\section{AUTOR}

DANIELA SZPILBARG

Universidad de Buenos Aires - CONICET 